\section{PERFUSION OF THE HEART WITH NICOTINE.}

BY D. T. BARRY, M.D.R.U.1., F.R.C.S. ENG., PRUFESSOR OF PBYSIOLOGY, UNITERSITY COLLEGE. CORK.

THE action of nicotine on the heart has been investigated by many workers, whose attention has chiefly been directed to the results of dropping the drug upon its surface in various concentrations or by injection into the circulation. A few well-known works bearing on this may be quoted from at the outset.

Pembrey and Phillips 1 state that injection of the drug into the circulation of a frog causes the heart to stop with the ventricle contracted and the auricles engorged with blood. Dropped on the heart in a 0.1 per cent. solution, these observers say that it causes slowing in two or three minutes, and when this occurs stimulation of the vagosympathetic nerve, which previously stopped the heart, is now incapable of causing arrest, while stimulation of the crescent still causes stoppage. This is interpreted as a paralysis of the pre-ganglionic fibres of the vagus, while the post-ganglionic fibres are unaffected.

Brodie ${ }^{2}$ shows a record in which slowing is caused by a weak solution in a few seconds. He states that the drug first stimulates and then paralyses nerve cells and strong doses paralyse nerve fibres.

Stirling "says that even with a full nicotine effect sinus stimulation causes acceleration due to excitation of accelerator fibres which have no cell in their course in the heart. There is no account of an appreciable stoppage of the heart nnder its influence, but permanent arrest by a drop or two of pure nicotine.

In the experiments here about to be recorded the frog's heart was systematically perfused with nicotine in varying strengths and a large number of observations made on more than one hundred hearts of Rana temporaria. Some hundreds of graphic records have been obtained.

\section{Method.}

The special cannula previously described by me ${ }^{4}$ was inserted into the vena cava below the liver as described by Mines. 5

The nicotine was mixed with Ringer's solution and neutralised with dilute sulphuric acid until the $\mathrm{CH}$ was about $1 \times 10^{-8}$, as shown by indicators. Neutralisation is important, as the degree of alkalinity of even a very weak solution of nicotine has a deleterious action.

The velocity of flow of the perfusing finid could be varied by altering the levels of the flasks by a rack and pinion adjustment, a range of 1 to 4 c.c. per minute being given, which was uniform in all three flasks.

The effects of stimulation of the vago-sympathetic nerve, of the intracranial vagus and of the sympathetic, independently, were tried carefully in many instances before, during, and after nicotine action. In this connexion it is necessary to mention that vago-sympathetic stimulation of ten gives a different result in the heart which is being perfused with Ringer's solution from that given before perfusion was begun. (Fig. 1.) The same applies to stimulation of the intracranial vagus. The lower the pressure of perfusion the greater is the resemblance between the pre- and post-perfusion stimulus. Attention has also been called to this by Ponirovski. ${ }^{6}$ Even the tension of the lever threads, especially in the case of the auricle, when no perfusion is taking place, tends to cause escape.

A standard stimulus was employed throughout, the secondary coil being fixed in one position and the primary current from a couple of Leclanché's cells regularly tested with a voltameter. Of 50 frogs examined during the month of March arrest of the heart beat, varying in duration from a few seconds to a minute, was elicited by a vago-sympathetic stimulus in 30. Many of these when perfused and attached to the levers exhibited merely some slowing as a resuit of the stimulus, but about half of them showed acceleration instead. (Fig. 1.) This record 7 shows that the vagosympathetic effect in the empty heart (the aortæe were cut) is much slowing, with a great fall of ventricle tone. The ventricle lever was raised between the arrows as it fell on to the signal marker. At the first gap in the tracing the

${ }^{1}$ Pembrey and Phillips: The Action of Drugs on the Heart. p. 58.

Brodie: Essentials of Experimental Physiology, p. 132

Stirling: Outlines of Practical Physiology, p. 404.

Barry: Tge LANCET, July ist, 1916, p. 14

Ponirovski : Publications of the Veterinary Inst.. Kbarkov, Jan. 13th, 1916

${ }^{7}$ All records read from left to right, the upper tracing being that of the auricle, the time of the drum movement being $1 \mathrm{~cm}$. per minute except where otherwise indicated cannula was inserted into the vein and perfusion begun. The third signal shows the changed response to the same stimulus-viz., a marked augmentor effect. The rest of the record will be referred to later.

After 10 or 15 minutes' perfusion the inhibitory effect is often restored or restored in part; at any rate, after this interval the response is pretty uniform.

\section{Action of Nicotine.}

It is considered by many that nicotine affects nerve cells chiefly, with little or no depressing action on nerve fibres. Stirling's ${ }^{s}$ observation that accelerator nerves are still active with a full nicotine effect suggests the question, What is meant by a full nicotine effect and how is it established? One or two minutes' perfusion with a strong solution, such as 0.5 or 1 per cent. of nicotine, will always cause complete arrest of the beat for several minutes, with loss of irritability of the muscle, and in this phase, of course, there is no contraction, not to mention acceleration, from a muscle stimulus. The stock of material for contraction is not altered in amount by drugs, as mentioned by Burridge. ${ }^{9}$ Nor is it diminished by 1 or 2 c.c. of a 0.5 per cent. of nicotine, so that the contractions return spontaneously in such a case, and when they do a stimulus. which, prior to nicotine action caused acceleration, now fails to do so, whether it be through nerve or muscle. (Fig. 2.) This record shows that even a 0.2 per cent. solution can depress accelerator fibres. Of course, it is possible that accelerator action here is merely counterbalanced by inhibitor stimulation at the same time, but this would apply to the prenicotine stimulus also. Stimulation of the sympathetic alone has been noticed often to be without effect on rate during nicotine action. First, acceleration caused by a vago-sympathetic stimulus, next the effect of the nicotine is shown, and after some recovery from this the same stimulus fails to cause any acceleration, which it does cause, however, again (third signal) on washing out the drug with Ringer's solution at $R$. Beyond raising the auricle lever slightly from the great sweeping movements of the lower one at the thick lines and stopping the drum at $\mathbf{S}$ and $\mathbf{R}$ for about half a minute nothing was changed throughout except the perfusing fluid.

The action of nicotine may be considered from different standpoints. Taking the two factors, (A) nerve and (B) muscle, the relative importance of which in cardiac phenomena has given rise to much discussion, the former exhibits inhibitory and excitor elements and the latter has to be subdivided into auricular and ventricular muscle and the muscle connecting the two, which for convenience is here referred to as the A.V. bundle. ${ }^{10}$ The effect of the drug, too, on the output of the heart in a certain phase of its action has been tested, and this effect is in the direction of increased work. The cardiac effects of the drug are certainly worthy of close attention, whether we consider them as applied specifically to the elucidation of the many secrets still remaining in the heart mechanism, practically all possible factors in which are influenced by it more or less independently, or as to a more general significance of the phenomena observed. The principal immediate effects of a 0.5 per cent. solution have been already stated, but attention has been chiefly directed in these experiments to weaker solutions-namely, 01 and 0.2 per cent. - which it will be well to consider first in a general way.

On a fresh heart the effects of a 01 per cent. solution of nicotine are somewhat as follows: Slowing of the rate sets in usually before the end of the first minute, with or without a previous acceleration; the auricle beat is increased in amplitude, while that of the ventricle is shortened and shows incomplete diastole. This weak solution if dropped on the heart while it is being perfused has very little effect on the rate and causes a very slight tonus or none. It requires a strength of nearly 1 per cent. to cause an appreciable stop when dropped on the Ringer perfused heart, while intermediate solutions of 0.2 or 0.3 per cent. used by dropping. generally cause some slowing and increase of auricle beat in like case.

Though the heart becomes inured to the drug in such a way as to recover much of its normal condition under its

$$
8 \text { Stirling : Loc. cit. }
$$

9 Burridge : Quart. Jour. Med., 9.43; $9271,1915,1916$.

10 In the anatomical sense the connecting muscle between auricle and ventricle in the 'rog's beart is not a bundle, but it is physiologically equivalent to the mammalian bundle, and the designation is adhered to because it is less confusing than other terms 
A

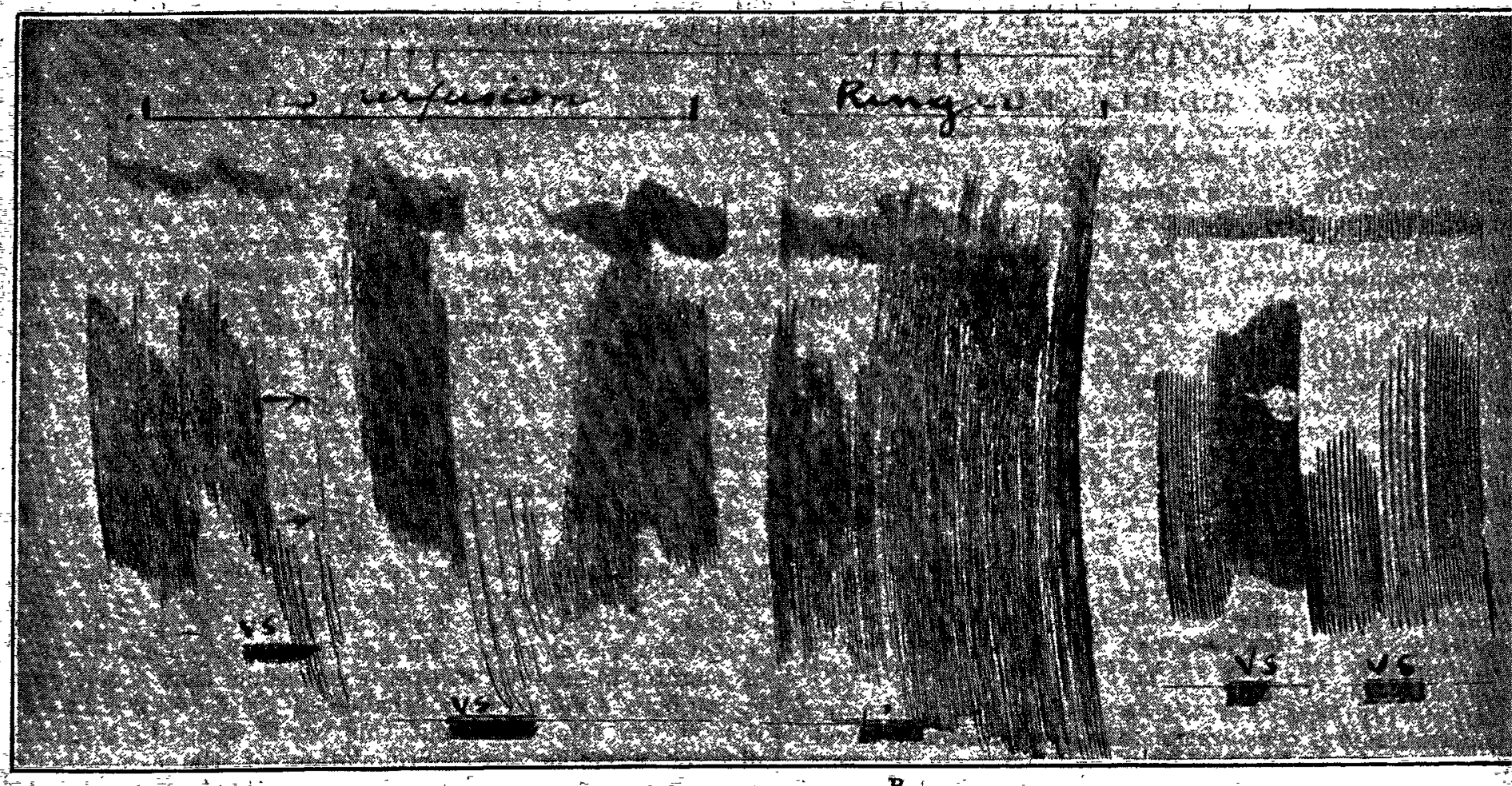

$\mathbf{B}$

zFig. 1. - Rffects of vago-symiathetic stimulation with and without simple perfusion. Slowing and losis of tone (first two signals) without perfusion. Augmentor effect with perfusion (third signal). The last two signals represent the same stimulus in recovery from nieotine.

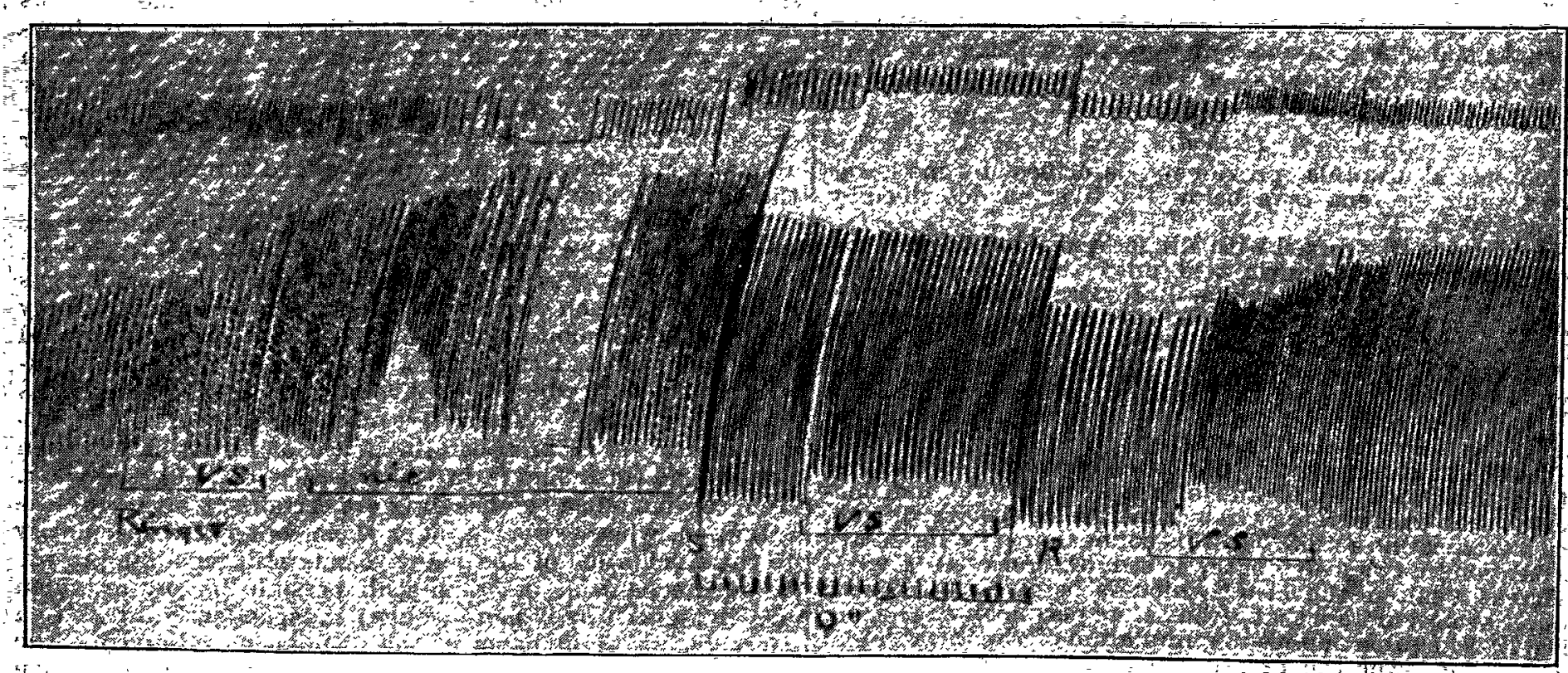

FIG. 2.-Vago-sȳmpathetic stimuli (V.s.) causing acceleration before and after nicotine effect, but not during this.

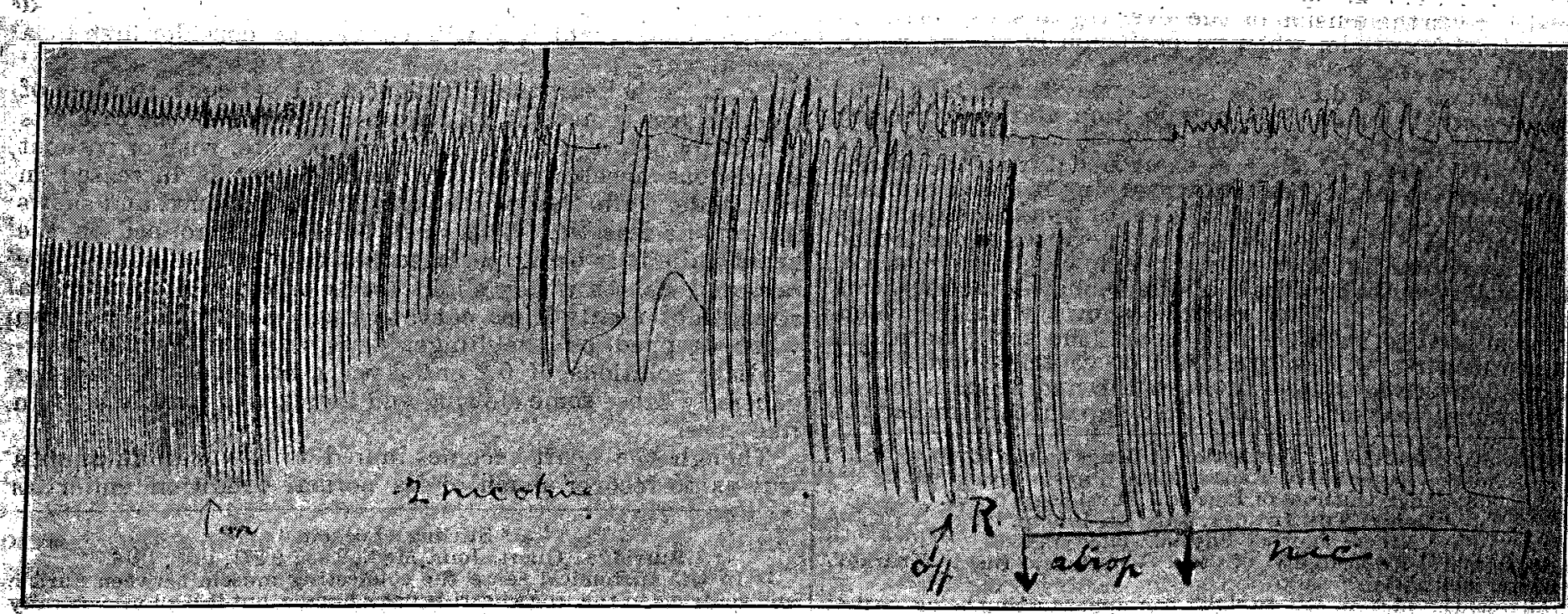

FIG. 3.-Effect of 0.2 per cent. nicotine before and after atropine. Strong atropine perfused about three minutes between arrows. Nicotine action as before, but less tonus. 
continued action, it does not always show an improvement on its pre-nicotined state; but it frequently shows for a varying time a larger beat if the nicotine be replaced by Ringer's solution after the first minute, and in this phase it is capable of performing increased work, as referred to above and as dealt with later on.

A 0.2 per cent. solution acts similarly to the above, but with a more pronounced effect. The steps of the action resemble those described for 0.1 per cent. up to a certain point - that is to say, slowing and increased tone of the ventricle-and after a while a complete cessation of beat. (Fig, 3.) The time of onset of this arrest varies from one to four minutes, and its duration with continued nicotine perfusion is from one to three minutes.

\section{A. Nerve.}

Acceleration before slowing is not invariable, but quite common with a 0.2 per cent. solution, and still more frequent with 0.5 per cent. If acceleration is observed once in a particular heart it occurs again and again in this with the same nicotine solution after a thorough washing out with Ringer's solution, and this consistency is also shown with the other phenomena, in degree, duration, time of onset, \&c. (Fig. 4.) Three arrests of the beat b 02 nicotine are shown after a preliminary acceleration and s lowi ng. The drum movement was stopped in each case soon after the beat ceased, and this was allowed to recover under the action of the alkaloid. Then a "step" of the recovered contractions to the mark $R$, where washing ont took place. The strong contractions at the end occur under continued nicotine perfusion.

Acceleration by nicotine has been noticed by other observers and has been attributed

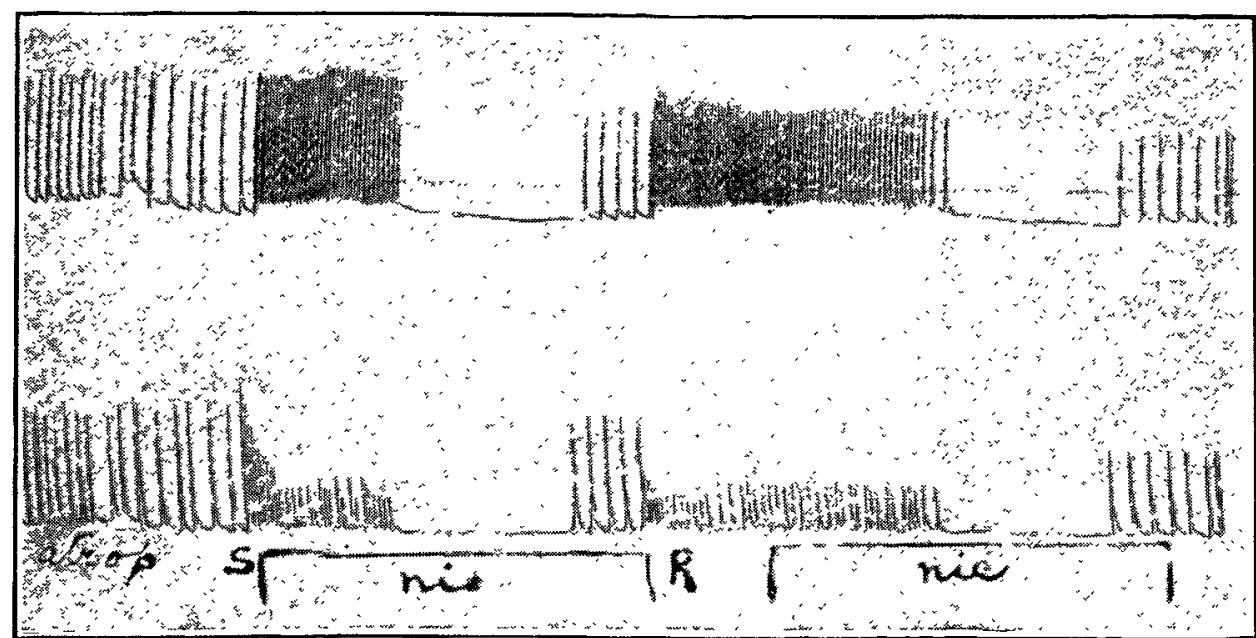

FIG. 5. - Irregular slow beat caused by strong atropine perfision which ceased at $\mathrm{s}$. Then 0.2 per cent nicotine stopped heart in two minutes. Same effect later when all atropine washed out (H).

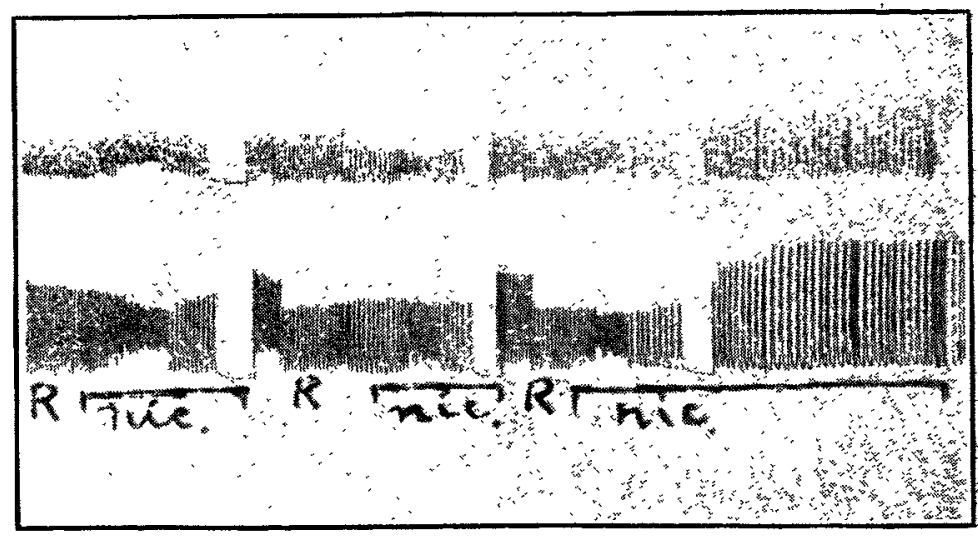

FIG. 4.-Tbree applications of 0.2 per cent. nicotine each after thorough Ringer washing at $R$. The accelerators are stimulated in each case. Drum stopped a few minutes at each break. either to the presence of sympathetic cells in the heart/mechanism is not quite satisfactory in the light of the (Cullis and Dixon ${ }^{11}$ ) or to its action on part of the neurone results about to be referred to.

11 Cullis and Dixon : Jour. of Physiol., 1911. xlii., 156.

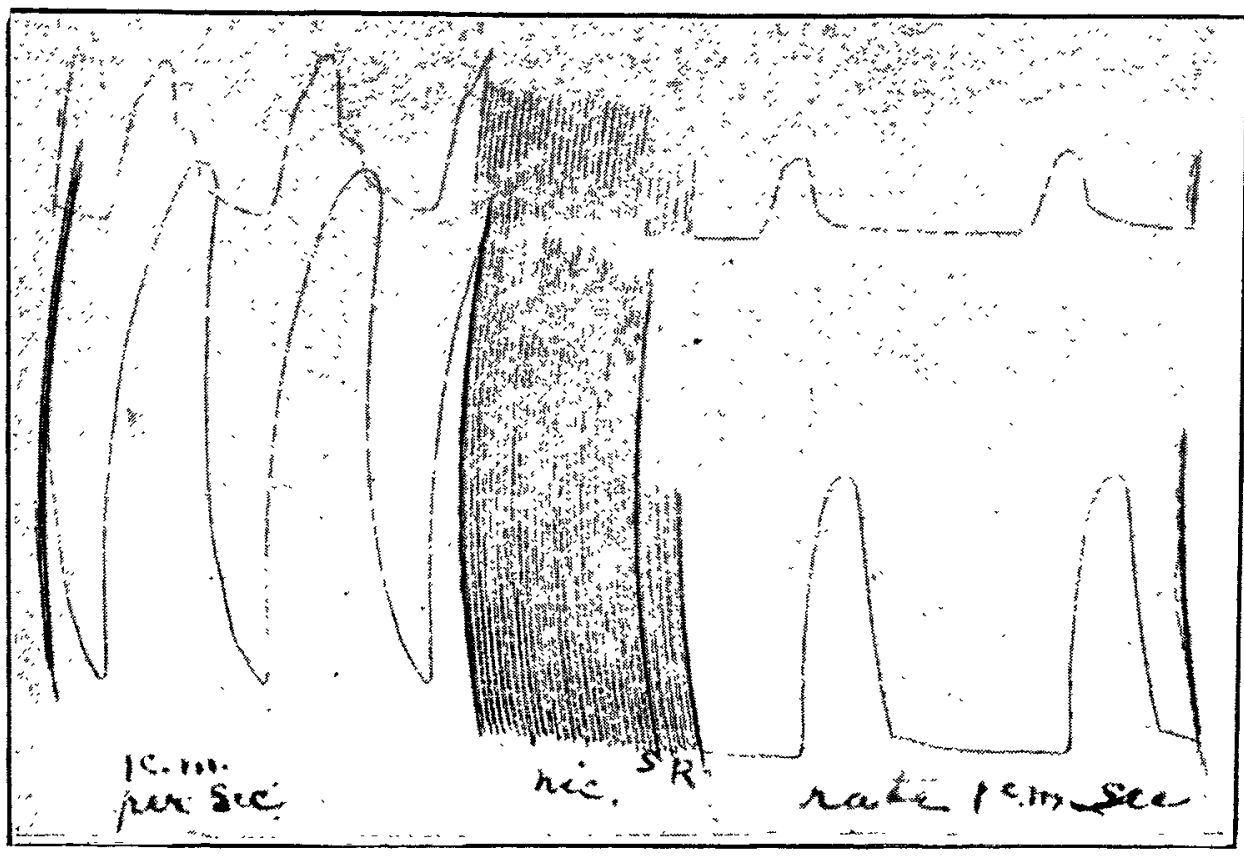

FIG. 6.-Augmentor effect with reversed beat. Normal beat at $R$.
If the inhibitory effect, slowing and cessation of beat for a time, is due entirely to action on ganglion cells or preganglionic vagus it should be abolished by something which could be relied upon to suppress vitality in these parts, such as atropine. It is said that they are paralysed in the heart by $0.001 \mathrm{mg}$ of this, and perfusion with atropine solutions representing this dose, administered in the course of $a$ few minutes, was tried as a preliminary to nicotine action, but without effect. Stronger solutions of atropine were next tried, and not until 005 per cent. solution was reached was any appre. ciable antidotal effect noted, beyond lengthening the time of onset of slowing. With this and stronger solutions (yielding about $5 \mathrm{mg}$. per minute or more) there is a depressing action on muscle. (Fig. 5.) The result in all cases of atropine perfusion was that it never prevented the slowing caused by nicotine. though in strong solution it sometimes diminished the degree of slowing as well as prolonging the time necessary for its onset. This nicotine effect is very like the nsual one, and

12 Dale and Laidlaw : Jour. of Physiol., 1910, xli., $\mathbf{1}$. 


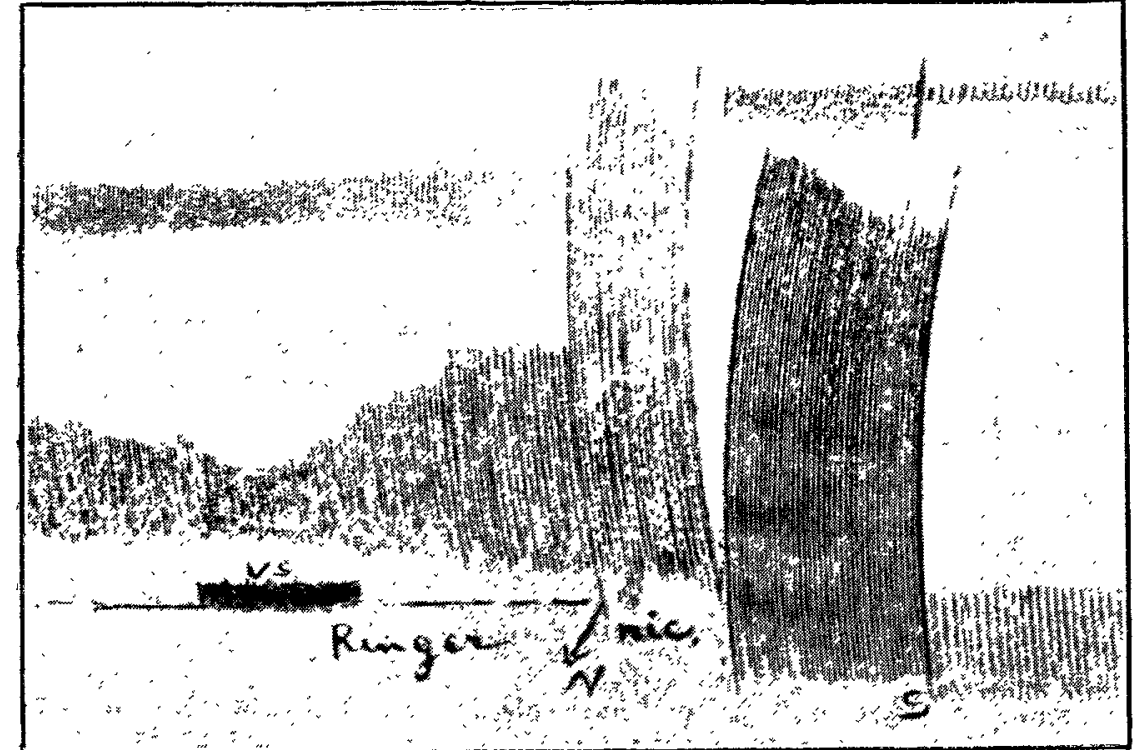

FIG. 7.-Great force of beat due to nicotine whe 1 applied after vago-sympathetic stimulation. Perfusion stopped half an hour at s.

suffers most with low perfusion pressure. Nicotine in this case stops the heart as before, and it resumes with increased pressure (nicotine + Ringer).

The slight counteracting effect of atropine may be due to the fact that nicotine excites both ganglion cells and post-ganglionic fibres, whereas atropine does not affect the latter. The modified nicosine response given in some instances after large doses of atropine may be accounted for by supposing that in strong solution atropine depresses post-ganglionic fibres without com. pletely abolishing irritability in them.

The purely augmentor fibres in the heart are also stimulated by nicotine, and this effect probably outlasts that on the other nerve elements. It is held in check or antagonised during the excited state of the inhibitory fibres, and if the latter condition is curtailed or prevented by turning on Ringer's solution before it is due to come on, the greatly increased force of the beat is seen. (Fig. 6.) It is also sometimes seen after the full effect of the nicotine solution, and may occur with a reversed rhythm - to be referred to directly. The record shows the reduction of the beat to normal after the slowing, if we take it that the ganglia bave been half an hour's delay at S (stationary drum). Augmentation paralysed, must be brought about through some other is enhanced and more quickly brought out if sympathetic channel. If, as has been shown, nicotine excites the post- tone is increased by stimulation beforehand. (Fig. 7.) In ganglionic fibres (accelerator) of the sympathetic, there is this case it was the vago-sympathetic trunk that was

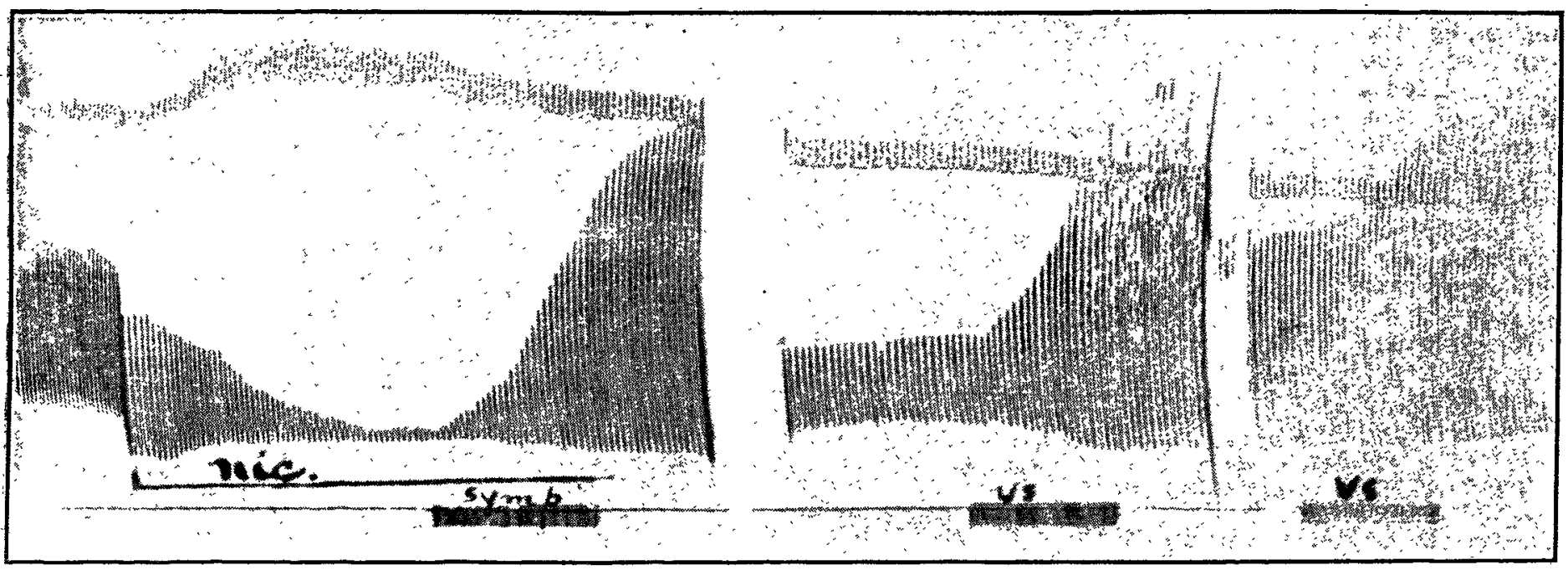

FIG. 8.-Failing ventricle in nicotine action restored by sympathetic stimulation; also strong augmentor effect of vago-sympathetic on ventricle. (Signals 2 and 3. )

no reason to suppose that the inhibitory post-ganglionic fibres : stimulated (signal) during Ringer perfusion; this causes are not also stimulated by it, whether we regard it as acceleration and some increased force. Half a minute's acting on nerve trunk proper or on receptive substance (Langley ${ }^{13}$ ).

Considering it well to leave no doubt of the failure of atropine to counteract nicotine slowing the former was perfused in still stronger solution, 0.1 per cent., until the muscle showed unmistakable signs of deterioration. These stronger solutions of atropine bring about a marked slowing of the heart and deterioration of the muscle metabolism, which is more marked in the ventricle, and augments this depressing effect of nicotine, but the slowing or stopping action of the latter is practically unaffected. (Figs, 3 and 5.) The latter shows a hypodynamic ventricle where perfusion with both drugs had been alternately performed for about an hour; a 0.2 per cent. nicotine stops the heart in about two minutes. This is again washed out at $R$, and again nicotine shows the same effect as previously. The depressing action can be intensified in the auricles by running a stout needle through them which establishes a leak, and this determines that the auricle muscle

\footnotetext{
13 Langley: Jour. of Physiol, 1906, xxxiii., 374.
}

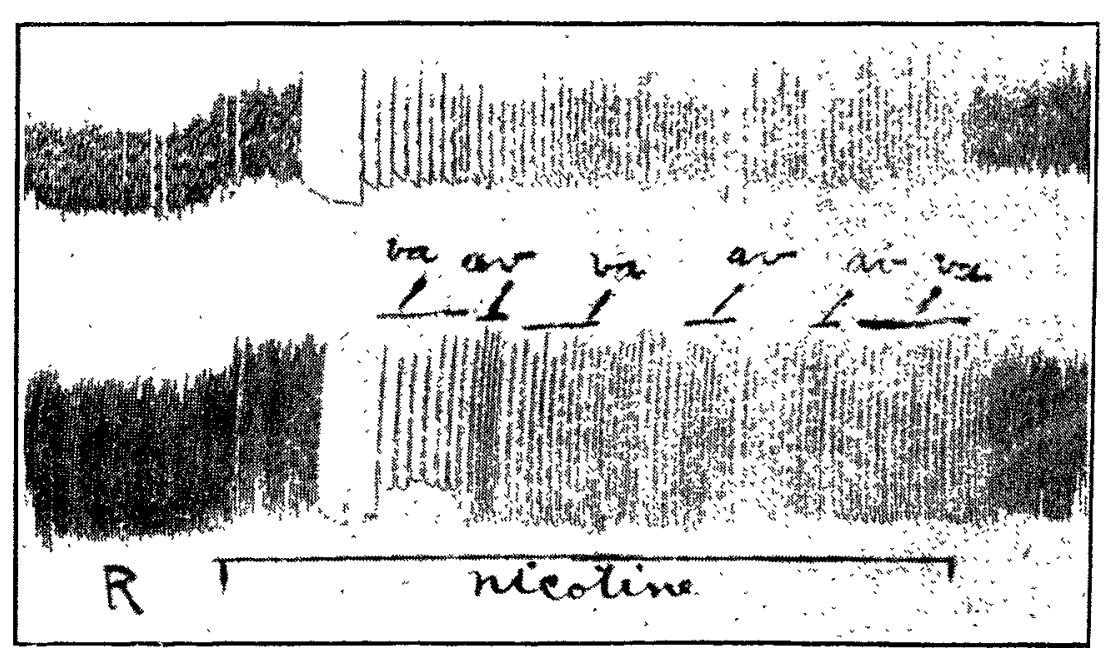

FrG. 9.-Alternation of A.v. and $\nabla$ A. rhythm during nicotine action. The auricle lears in the fast groups and the ventricle in slow portions. 


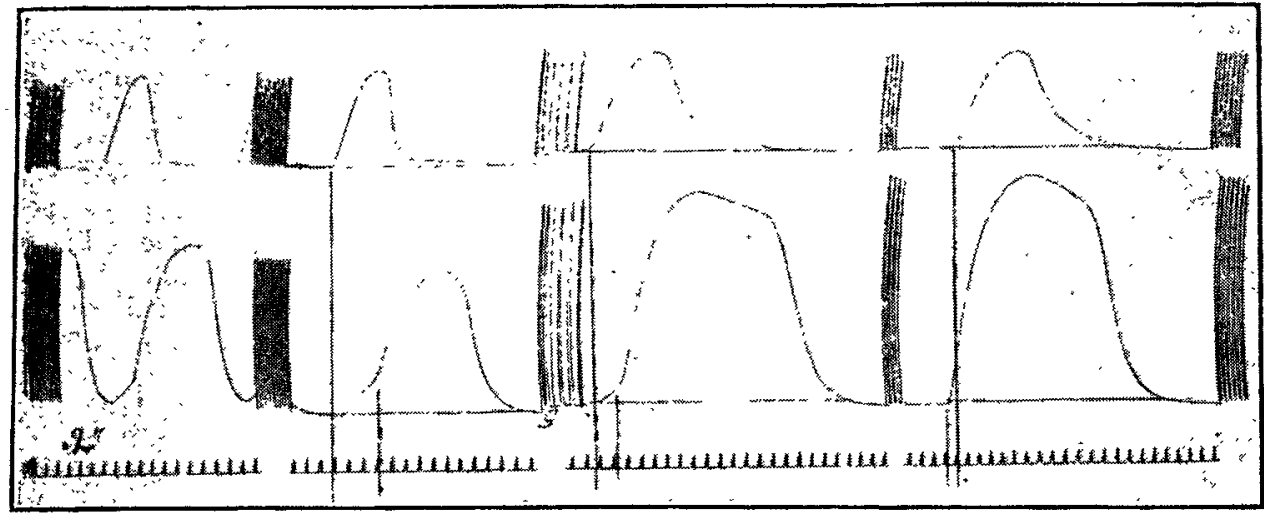

FIG. 10.-Reversal of rbythm by nicotine. The A.v. interval is seen to be shortened before the vertricle takes up the lead in the cycle.

adjusted at the gap in the record, where a portion was cut out, a further step is shown, and finally the return to a low condition without perfusion which had been stopped for about half an hour at the point $S$. The inhibitory elements are, of course, stimulated by the electric current as well as the augmentor, but we may suppose from the result that the balance of increased tone is with the latter, and the nicotine effect on this is more marked than on the other.

This phase of nicotine action resembles rather the effect of adrenalin in the great size and force of beat than that of digitalis or veratrine. In it the rate may be fast-viz., 60 per ninute--or comparatively slow, half-rhythm, irregularity, \&c. The effect of a failure of output by the ventricle is well shown by the auricular tension. The first signal represents stimulation of the sympathetic nerve. A few inches of the tracing which were without interest were cut out at the gap and the vagosympathetic this time stimulated (second signal) some ten minutes after the sympathetic (the sympathetic stimulus was applied below the vagus ganglion; the vagosympathetic stimulus to the trunk of the nerve high up). Again we have a pure augmentor effect which is repeated ten minutes later on (third signal). The effect is confined to the ventricle, and points to independent augmentor impulses to that chamber. So in this, after nearly an hour of perfusion with a 0.1 per cent. solution of nicotine, the augmentor fibres to the heart are still active. The period of depression in them supervenes sooner or later, as in the other elements. There may be a temporary depression which is quickly recovered from even under continued action of weak nicotine, or there may be no depression period with a weak solution until after prolonged action. As already shown, there is evidence of accelerator depression by 0.2 per cent. nicotine (Fig. 2), and it is evident that there is no augmentor effect of stimulation at this time either.

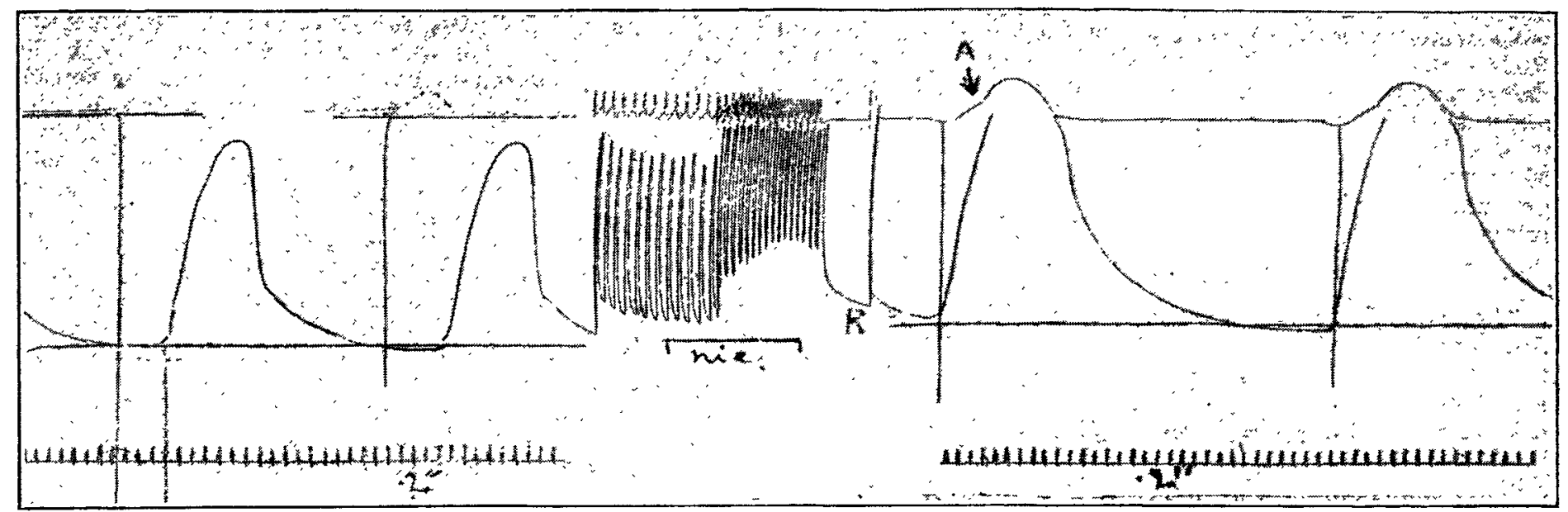

FIG. 11.-Reversed ruythm quickly established in accelerator phase of nicotine. Ringer solution was perfused for a few seconds to reduce tonus at $\mathrm{R}$. A beat begins at arrow.

about 15 to 20 per minute. The accelerators are quickly stimulated (pure acceleration is of ten the first sign of the drug effect) and recover quickly from succeeding depression; they recover sooner than the inhibitors. (Fig. 1.) The last two signals (Fig. 1) show stimulation of the vagosympathetic nerve with an interval of two minutes or less in recovery from perfusion with 0.2 per cent nicotine. Acceleration in the first of these is shown changed to slowing and increased force in the second, but only in the ventricle. During the acceleration response the inhibitor elements were still in the depressed stage, but two minutes later they have recovered sufficiently, not only to prevent an accelerator effect, but to cause some inhibition at least in the ventricle.

The augmentor fibres may remain active when the accelerators seem to be out of action in continued perfusion, and even when the muscle metabolism shows marked signs of deterioration. (Fig. 8.) In the first part of the tracing the ventricle beat is falling off quickly, which is one way of showing its depressed metabolism; other ways in which this is shown are the alternating pulse,
This greatly increased force of the beat suggested a test of the amount of work done by the heart and how far it might be increased by nicotine stimulation. The method adopted was a very simple one.

A glass tube 40 inches long, of about $4 \mathrm{~mm}$. diameter, was drawn out to a cannula at one end, and this was inserted into the left aorta of the frog, while the right was tied off or clamped. The tube was then placed in a nearly vertical position and the fluid expelled by the ventricle mounted up its interior in a series of intermittent movements according to systoles. Te systoles. Testing the normal heart on Ringer's solution
with a supply of $3.5 \mathrm{ccc}$. per minute, the fuid rose to a height

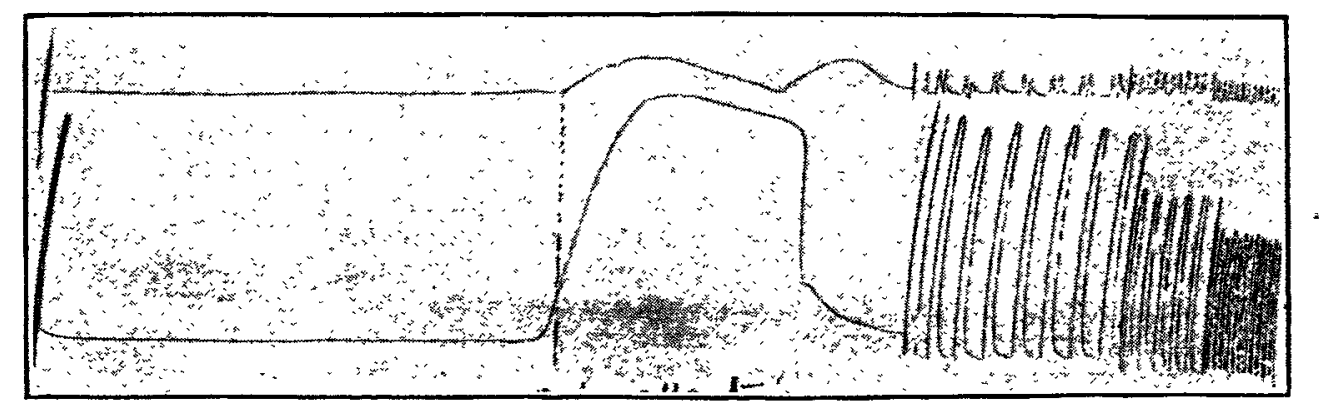

$\therefore$ :FIG. 12.-Donble auricle beat in rerersed rhythm. Description in text. 
(vertical) of 20 inches on an average, beyond which the heart failed to send it. Lowering the perfusion bottle to relieve the auricular stress and to give the heart a rest did not alter the fluid in the tube, which was kept up by the aortic valve. On further trial no increased work was done, but now, after lowering the perfusion bottle to relieve once more, the Ringer's solution was shut off and 0.2 per cent. of nicotine solution turned on for half a minute with the same feed. As a result of this the heart-beats were much augmented and the fluid was soon driven higher, with greatly increased strokes, until it passed right over the top (vertical height over 30 inches). This result was obtained frequently.

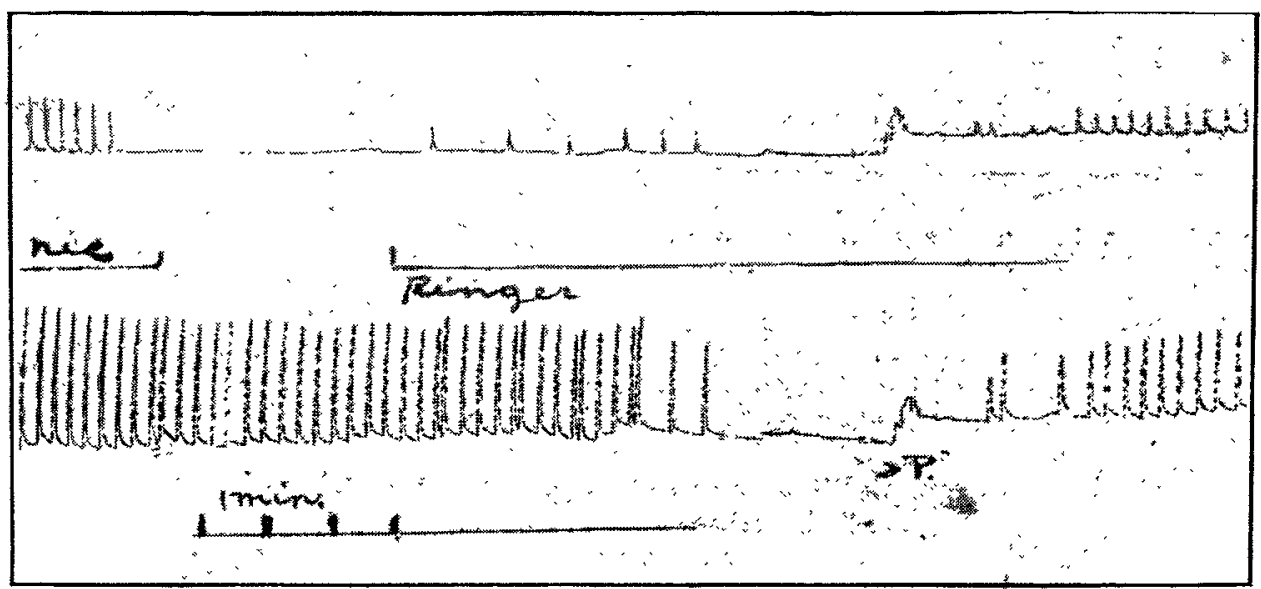

FIG. 13.-Independent ventricle rhythm. Recovering auricle causes extrasystoles. After fuur of these the independent contractions in ventricle suppressed.

A very high pressure is without ill effect on the heart after this dose of nicotine, as may be seen by the ease with which it deals with an increased feed like that of Ringer plus nicotine. Instead of cutting off the nicotine in half a minute, the clip may be removed from the Ringer tube and this solution allowed to flow with the former. Indeed, it has been frequently noted that the slowing effect of nicotine, 0.2 per cent., can be greatly diminished by raising gradually the perfusion pressure in this way after some half minute even without dilution by Ringer's fluid.

\section{B. Musole.}

The great force of beat has to be attributed in part to a direct action of nicotine on muscle fibre. Langley ${ }^{14}$ showed that skeletal muscle was stimulated by it when all nerves had degenerated. In this as in nerve it first excites and then depresses, but even in the state of depression, brought about by itself, nicotine is capable of temporary stimulation of muscle. Nicotine depression of muscle, too, is thoroughly independence of nerve, and the stock runs down when nerve influence has been long removed.

Generally speaking, ventricular muscle is more susceptible to the influence of nicotine than auricular, and shows the first signs of defective metabolism, as well as the more marked response (tonus and increase of beat) to the exciting action. The auricles and ventricle sometimes recover from nicotine stop at different times, the auricles, for instance, resuming their beat some time before the ventricle. But we can determine a stronger action on the auricle by perforating as before mentioned, so that its muscle and nerves are more affected and the ventricle beats independently. In such a case the auricle beat when resumed nearly always follows the ventricle-that is, a reversed rhythm. This reversal, too, frequently occurs in recovery from nicotine stop without perforation of the auricles, and in the progress of recovery there is often a struggle between the two chambers for the lead. (Fig. 9.) The accelerated patches in the record are those where the rhythm is A.V., and the slower those of the V.A. sequence. At the change from the former to the latter an auricle beat is missed and sometimes two. With reversal, too, the auricle may cease to beat altogether for a time, while the ventricle beats alone. Reversal is more common when nicotine perfusion is kept up than when replaced during the stop by Ringer.

There is no difficulty in detecting the last-mentioned changes with the eye, whether it be directed to the lever points or to the chambers themselves, but by spreading out the contractions on a fast drum it can be shown graphically. (Fig. 10.) Shortening of the A.V. interval is also a feature of nicotine action and can be noticed preceding the change to V.A. rhythm. This change can occur without pushing nicotine to the arrest of the heart beat. At first an ordinary A.V. contraction is shown (Fig. 10) with an interval of $3 / 5 \mathrm{th}$ second. At $S$ nicotine was perfused for half a minute and about one minute later the A.V, interval is $1 / 5$ th second. This does not occur in the acceleration phase but with slowing. Two minutes later still the reversed rhythm is shown. It can also be seen in the acceleration stage before any slowing is caused as the ventricle tonus passes off with a few seconds' Ringer perfusion. (Fig. 11.) A return to the normal A.V.

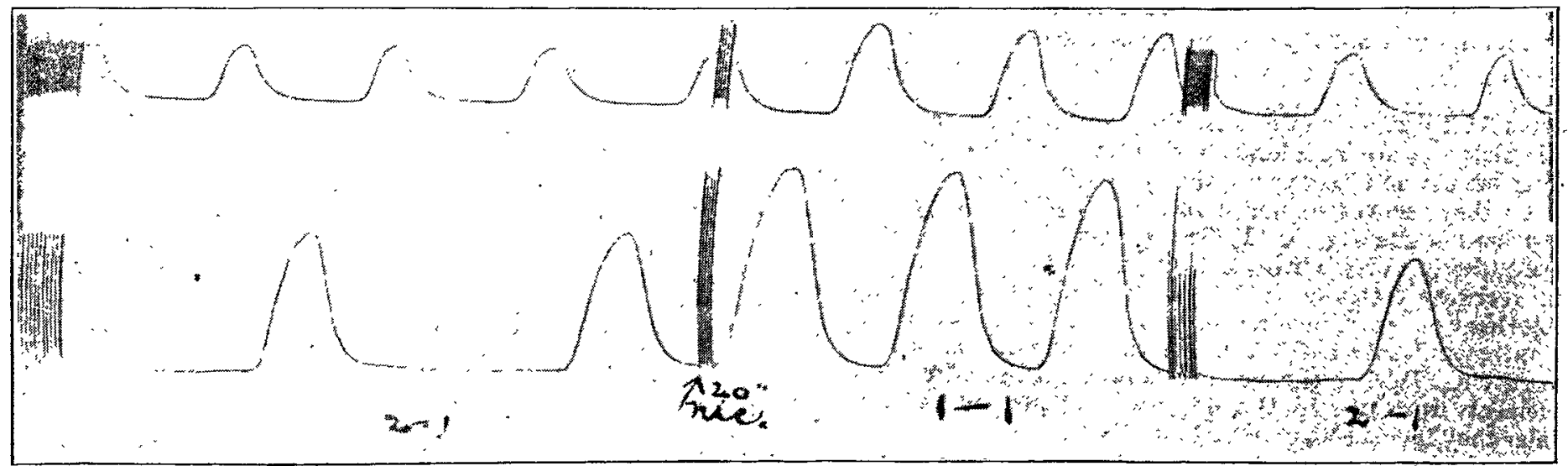

FIG. 14,-Block removed temporarily by nicotine. Central part of nicotine shows a 1;1 rhythm.

recovered from if due to no stronger concentration than that of 0.2 per cent., even when this has acted over quite long periods, and it cannot be said to affect the life of muscle as judged by the duration of its vitality. Carlson ${ }^{15}$ remarked that a 1 in 50,000 solution of nicotine depressed the muscle of the heart of limulus. But the nicotined frog heart can go on beating for several days in favourable conditions. Rhythmicity of heart muscle has probably but a limited

14 Langley: Lnc. cit.

15 Carlson: Amer. Jour, of Physiol., 1906, xvii., 177 rhythm always occurs with a few minutes' Ringer perfusion.

A phenomenon which has been frequently observed in this reversal is a double beat in the auricle (Fig. 12), the second beat occurring at the height of ventricular systole and during the refractory period of the latter. The fast movement of drum shows the beat first to be reversed. Perfusion with Ringer's solution for one minute does not alter the rhythm (it is still V.A.), but it changes the appearance of the auricle tracing. Two minutes' perfusion at the next stop 
gives the ordinary rhythm (A.V.). The significance of this double auricle beat is that the first is dependent on ventricular contraction which has been established as leading the cycle by some influence of the drug on its ordinarily subordinate mechauism. The higher mechanism, ordinarily predominant, is now recovering, and the second beat is an indication of this ; the stimulus from this second beat falls during the long refractory period of the ventricle and fails to excite it. The condition is interesting from its bearing on de Boer's ${ }^{16}$ work, who attributes to this long refractive phase the chief rôle in bringing about halved rhythm with veratrine, \&c., while the view of Engelmann ${ }^{1 \tau}$ and others is that the halved rhythm is caused by lowered condactivity from auricle to ventricle.

As a contrast to this we may examine another state where the ventricle is beating independently of the auricle, the latter being brought to a standstill, as already explained. (Fig. 13.) Here the recovery of the auricle takes place. without reversal-that is, independently of the ventricle mechanism, which is not strong enough to determine it. The auricle beats, in this case occurring after the refractory phase of the ventricle, cause extrasystoles in the latter, until it takes up its normal secondary habit of following the auricle, as shown by the last two beats before the interval. The heart then ceased to beat, probably because the perfusion pressure was low; when this was raised at $P$ the ordinary A.V. rbythm was established in a couple of minutes. The last of the extrasystoles is followed by a longer compensatory pause than the others, and during this the auricle assumes command, and this to the extent of stopping the ventricle with it when it ceases to beat. This group of auricle beats is characteristic of recovery when cessation has occurred in either chamber, isolated contractions taking place irregularly before ordinary rhythm is reestablished. The last two ventricle beats before the stop are a continuation of the extra series, the automatic contractions being suppressed.

A balved rhythm can be temporarily changed to 1-1 by nicotine in lowered ventricle metabolism (Fig. 14), and this may be interpreted in many ways. The drug may increase the conductivity of the connecting muscle (dromotropic), and thus break down a block as it is generally understood, or it may increase the irritability of the ventricle (bathmotropic or inotropic), though the refractory period with larger beats would tend to increased length. The stimulus from the auricle, too, is stronger because of the stronger contraction.

That the second beat in the auricle may possibly be caused by closure of the electric change accompanying the ventricle contraction has to be considered, though the explanation given is the more likely one.

Reversal of the heart rhythm in the turtle has been caused by Laurens and Gault. ${ }^{\text {is }}$ Conjoint stimulation of the vagus, which is separate from the sympathetic in the neck, and of the funnel connecting auricle and ventricle led to this V.A beat. Stimulation of the sympathetic changed this back to the normal rhythm. There was no change of rate, but the force of auricular contraction was less.

In the nicotine reversal in the frog stimulation of the sympathetic seems to be without effect in converting to normal ; it was certainly without immediate effect when tried in a few cases. We may suppose that the nicotine stimulation to predominance of the usually dependent mechanism of the ventricle is at least in part due to its stimulating action on ventricle sympathetic elements exceeding that on the auricle, while the inhibitory effect would be more potent in the latter. As already remarked, there is evidence of independence of action of ventricle augmentor elements. (Fig. 8.)

Schlomovitz and Chase ${ }^{19}$ tried to abolish the automaticity of the sino-auricular pacemaker in the turtle with nicotine and atropine, but did not succeed, and for this reason they concluded it was independent of nerve. There is no doubt, however, that nicotine causes a reversal of rhythm as here described. It has been seen many times.

As already stated, the A.T. interval is shortened in certain phases of nicotine action. If this interval is due to delayed conductivity of the contraction wave in the inter-

$$
16 \text { De Boer: Quar. Jour Exp. Pnysinl, x.. 4, p. } 383 .
$$$$
17 \text { Engelmann. Arch. fur d ges. Phrsiol } 1897,1 \mathrm{xv}, 108
$$

18 Laureris and Gault. Proc Soc Hxp. Biol. Med., 1916. xiii,

19 Schlomovitz and Chase : Amer. Jour. of Physiol., 1916 xli., 11 ? mediate region then nicotine shortens it by increasing the conductivity. It is likely that the drug shortens the latent period of ventricular muscle, but this has not as yet been verified.

\section{Conolusions.}

Nicotine in weak solution, 01 and 0.2 per cent, which has been neutralised, perfused through the hearts of frogs gives results which are fairly uniform, though certain variations occur.

The first eflect in a majority of instances is pure acceleration of the beat which is usually seen in less than half a minute from the introdnction of the drug. This gives way to slowing, a phase in which increased force of the contractions is also seen. When acceleration is not in evidence increased force is usually exhibited alone within the first half a minute, or both may occur together. The stimulation of accelerator fibres is soon followed by depression, and this double action is believed to be true of nicotine on the different elements responding to it.

Slowing of the beat leads to a definite stop lasting one or a few minutes, but this is not common with solutions of less than 0.2 per cent. After a period of quiescence the beart resumes its beat with a rhythm which may be irregular or regular, the amplitude of contraction being usually greater than before. The contractions are now of ten reversed-viz., apex to base sequence, and this is more comron after stronger solutions of nicotine, 05 to 1 per cent.

With 0.5 to 1 per cent. solutions cessation of beat occurs sooner than with weaker solutions, and lasts longer. With a solution of less than 1 er cent, it resumes beating spontaneously after arrest, with nicotine perfusion kept up, though this is not always so.

The slowing of the beat and arrest for a while is due to stimulation of the vagus endings. The failure of atropine to counteract this points to stimulation of post-ganglionic fibres. This resembles its effect on the sympathetic side causing acceleration.

The augmentor fibres are also stimulated by nicotine, and the succeeding depression is short-lived or may be. much delayed. Evidence is given of their activity in certain stages of nicotine effect. when inhibitor and purely accelerator factors seem to be suppressed. The activity of augmentor fibres to the ventricle seems to be possessed of a certain degree of independence, in consequence of which they may constitute the chief factor in independent ventricular rhythm.

The work done by the heart can be increased by nicotine stimulation. This is in part nervous and in part muscular in origin.

The delay in the passage of the contraction wave from auricles to ventriole is reduced by nicotine, in some instances independently of acceleration, and is frequently seen to lead to a reversed rhythm. The usually subordinate ventricle mechanism is stimulated to predominance in the cycle.

Though all of the phenomena mentioned have been observed many times, the only one which may be said to be unfailing in its appearance is slowing and arrest of the beat in proportion to the strength of nicotine used. Very strong doses of atropine do not abolish this.

Cork.

The rate Mr. Frederick E. Fenton.-Mr. Fenton, who died at his residence, Uxbridge-road, Ealing, on Uct. 16th, aged 64, was born in Chelsea, and received his medical education at St. George's Hospital and in Paris. He qualified in 1878, and later took the Fellowship of the Royal College of Surgeons and the Membership of the Royal College of Physicians of Edinburgh. He commenced practice in Ealing at a time when that place still retained some of the characteristics of a Middlesex village in the neighbourbood of London. By his all-round ability he soon gained for himself a good position professionally and the esteem of his neighbours. He was prominently associated with the Cottage Hospital at Ealing, and later became surgeon to the Western Skin Hospital and to the King Edward Memorial Hospital at Ealing, being later appointed consulting surgeon. Among his other active interests was that of the Volunteer movement, and he served in the Queen Victoria Rifles for 22 years, retiring with the rank of Surgeon Lieutenant-Colonel, with the Yolunteer Decoration. A good French scholar, he translated from the French A. Duval's "Artistic Anatomy" He leaves a widow, a daughter, and a son. who is a mernber of the medica! profession. 